\title{
豆科固氮植物对 $\mathrm{CO}_{2}$ 加富的生理响应
}

\author{
牛书丽 蒋高明* \\ (中国科学院植物研究所植被数量生态学重点实验室, 北京 100093)
}

\begin{abstract}
摘 要 全球 $\mathrm{CO}_{2}$ 浓度升高对植物的影响成为近代植物生理生态学研究的热点, 豆科植物因其独特的固氮能力使 其研究具有重要价值。从光合生理、固氮特性及碳、氮代谢等几个方面综述了 $\mathrm{CO}_{2}$ 浓度升高对豆科植物生理生态 特性的影响, 总结出如下结论: 在高 $\mathrm{CO}_{2}$ 浓度条件下, 豆科植物的光合速率、根瘤量和固氮能力均得到提高, 光合和 固氮之间的关系更加紧密, 两者互为因果, 相互促进, 保证了高 $\mathrm{CO}_{2}$ 浓度下植物体内碳、氮代谢的平衡、协调进行。 关键词 豆科植物 光合速率 根瘤 固氮活性 $\mathrm{C} / \mathrm{N}$ 比
\end{abstract}

\section{EFFECT OF ELEVATED $\mathrm{CO}_{2}$ ON LEGUME PLANTS WITH NITROGEN FIXATION}

\author{
NIU Shu-Li and JIANG Gao-Ming * \\ ( Laboratory of Quantitative Vegetation Ecology, Institute of Botany, the Chinese Academy of Sciences, Beijing 100093, China)
}

\begin{abstract}
It is well known that the global atmospheric $\mathrm{CO}_{2}$ concentration, presently about $360 \mathrm{ppm}$, is increasing and is expected to double by the end of the next century. An increase in the atmospheric $\mathrm{CO}_{2}$ concentration should increase the plant photosynthetic rate. However, the response strength of plants to elevated $\mathrm{CO}_{2}$ is dependent on the availability of nutrients, especially nitrogen. An increased $\mathrm{N}$ demand will result under elevated $\mathrm{CO}_{2}$ in a larger $\mathrm{N}$-sink of the whole grassland ecosystem. One way to maintain the balance between the carbon and nitrogen cycles in elevated $\mathrm{CO}_{2}$ would be to increase $\mathrm{N}$-import to the grassland ecosystem through symbiotic $\mathrm{N}_{2}$ fixation. So under the elevated $\mathrm{CO}_{2}$ conditions, legumes with $\mathrm{N}_{2}$ fixing ability should be much more important in the ecosystem, and studies on their response to elevated $\mathrm{CO}_{2}$ are significant.
\end{abstract}

The current atmospheric $\mathrm{CO}_{2}$ concentration is insufficient to substrata Rubisco in $\mathrm{C}_{3}$ plants. So, an increase in the availability of this substrate results in a rise in leaf photosynthetic rates. Compared with nonlegumes, legumes will increase much more in the natural ecosystem because of its $\mathrm{N}$ fixing ability. The authors propose three hypotheses to explain the stronger response of legumes to elevated $\mathrm{CO}_{2}$. First, nodules as a carbon consuming pool will stimulate the photosynthesis. Second, symbiotic $\mathrm{N}$ fixing increased the legumes leaf nitrogen content, which was positively correlated with photosynthetic rate. Third, lower $\mathrm{C}: \mathrm{N}$ in leaves of legumes than non-legumes under elevated $\mathrm{CO}_{2}$ promotes the decomposition rate of organic matter, which facilitates plant photosynthesis and growths.

Many studies showed that not only the number and the individual weight of nodules, but the nitrogenase activity per unit dry weight in legumes would be enhanced under elevated $\mathrm{CO}_{2}$. In addition, this enhancement of $\mathrm{N}_{2}$ fixation will result in a large $\mathrm{N}$-input to the grassland ecosystem. However, there has been disagreement as to the real causes of this increasing $\mathrm{N}$ fixing. Someone believes that it is because of specific nitrogenase activity (SNA) while others think that total nitrogenase activity (TNA) works. Mr. Bai gave reasonable explanation in the text. The increased $\mathrm{N}$ fixing is partly due to the increased $\mathrm{CO}_{2}$ assimilation under elevated $\mathrm{CO}_{2}$ because up to a third of photosynthates may be used for energy, reductants and carbon skeletons for nitrogen fixation and assimilation in annual legumes. Increased photosynthates delivered to nodules meet the $\mathrm{N}_{2}$ fixing needs. At the same time, the increased nitrogen content in legumes will also meet the increased $\mathrm{N}$ demand of photosynthesis and stimulated it.

In summary, it is likely that $\mathrm{N}$ content of $\mathrm{CO}_{2}$ enrichment of grassland ecosystem will increase through the increased symbiotic N-fixing ability, to match the increased photosynthetic potential offered by elevated $\mathrm{CO}_{2}$ concentration. There are facilitative interactions between carbon and nitrogen metabolism in legumes and they interact even more intimately under $\mathrm{CO}_{2}$ enrichment. Long-term studies are still needed to understand the biochemical mechanism of this response and the research material should be extended to field species.

Key words Legume plant, Photosynthetic rates, Nodules, N-fixing activity, C/N ratio 
人类活动已使大气中的 $\mathrm{CO}_{2}$ 浓度由产业革命前 的 $280 \mu \mathrm{mol} \cdot \mathrm{mol}^{-1}$ 增至目前的 $350 \mu \mathrm{mol} \cdot \mathrm{mol}^{-1}$ 左右 （Genthon et al., 1987; Carter, 1996), 且有加速增长 的趋势, 引起了广泛的关注。植物体对 $\mathrm{CO}_{2}$ 浓度升 高的最直接生理反应是植物光合速率的提高, 但不 同的植物对 $\mathrm{CO}_{2}$ 浓度增加的响应程度不同 (Alberto et al.，1996; Chen et al.，1996), 这样就造成了植物 间原有竞争关系的改变和植物群落结构的变化。豆 科植物是仅次于禾本科和菊科的第三大科，每年豆 科植物的固氮量几乎与工业固氮量相等(于振文, 1993 ), 其在自然界的广泛分布和其独特的自生固氮 能力决定了其在生态系统中具有举足轻重的地位。 研究豆科植物对 $\mathrm{CO}_{2}$ 浓度升高的响应不仅可以预计 未来全球变化条件下豆科植物的生长状况和它在植 被中的地位,而且还可以充分发挥其自生固氮特性 来调节自然生态系统和大气中的氮素平衡。

$1 \mathrm{CO}_{2}$ 浓度升高对豆科植物光合生理的影 响

$\mathrm{CO}_{2}$ 是植物光合作用的底物, 且目前大气中的 $\mathrm{CO}_{2}$ 浓度是 $\mathrm{C}_{3}$ 植物光合作用的限制因子, 因此, 人们 对未来高 $\mathrm{CO}_{2}$ 浓度下植物 (尤其是 $\mathrm{C}_{3}$ 植物) 的光合 作用变化进行了广泛的研究 (Sage et al., 1989)。在 对豆科植物的研究中大多集中于大豆 (Glycine max)、苜宿 (Medicago sativa) 和白三叶 (Trifolium repens) 等几种典型植物上, 其中对大豆的研究开展 的较多、较早 (Brun \& Cooper, 1967; Clough et al., 1981; Allen \& Boote, 1987), 其结论比较一致: 随 $\mathrm{CO}_{2}$ 浓度的升高, 叶片光合速率和水分利用效率均得以 增强。William 等 (1988) 指出生长在高 $\mathrm{CO}_{2}$ 浓度 $\left(660 \mu \mathrm{mol} \cdot \mathrm{mol}^{-1}\right)$ 条件下的大豆, 其叶片水平光合速 率比生长在 $330 \mu \mathrm{mol} \cdot \mathrm{mol}^{-1} \mathrm{CO}_{2}$ 条件下光合速率高 $50 \%$, 从而使豆荚产量提高了 $44 \%$ 。对紫花苜宿的 研究结果表明 $\mathrm{CO}_{2}$ 倍增组的表观光合速率比对照组 可提高 $18.7 \%$, 气孔导度略有下降 ( $12 \%)$, 蒸腾速 率减少了 $2.7 \%$, 水分利用效率提高了 $30.1 \%$ (项斌 等, 1996)。张其德等(1996)更深一步的研究了 $\mathrm{CO}_{2}$ 加富对紫花苜宿光合作用原初光能转换的影响, 认 为在 $\mathrm{CO}_{2}$ 加富条件下生长的紫花苜宿, 其叶绿体对 光能有更强的吸收能力, 可促进紫花苜宿叶片 PS II (光系统 II ) 原初光能转换效率、PS II 潜在活性、PS II 电子传递量子产量以及 PS I 活化能力的提高, 增加 荧光光化学猝灭组分, 降低非光化学猝灭组分。

叶片进行的光合作用, 是 RuBPcase(二磷酸核酮
糖羒化酶)活动的结果, 它受到 $\mathrm{CO}_{2}$ 浓度、温度和其 它环境因子的影响。目前空气中的 $\mathrm{CO}_{2}$ 浓度还不能 使 $\mathrm{C}_{3}$ 植物 RuBPcase 得以饱和。在高 $\mathrm{CO}_{2}$ 浓度条件 下, 因为增加了 RuBPcase 羧化方向上的底物浓度 (Webber et al., 1995), 使得通常可消耗 $C_{3}$ 植物初 始固定 $\mathrm{CO}_{2}$ 的 $25 \%$ 的光呼吸得到了抑制 (Ramachandra et al., 1993; Drake et al., 1997), 从而节约大量 的同化产物。但 $\mathrm{CO}_{2}$ 浓度增加条件下 RuBPcase 的 活性并没有得到提高( William et al . , 1988)。Vu 和 Allen( 1997) 试验表明: 高温、高 $\mathrm{CO}_{2}$ 浓度对大豆的 RuBPcase 蛋白含量有降低效应, 但与水稻相比, 这种 降低效应要小得多。这可能因为水稻叶片上的 RuBPcase 蛋白含量比大豆更易受到高 $\mathrm{CO}_{2}$ 浓度的负 调节, 因为高的 $\mathrm{CO}_{2}$ 浓度可以从蛋白含量和酶活力 下降两个方面来对 RuBPcase 进行负调节。

光合速率的增加引起单位面积生物量和产量的 增加 (Allen \& Boote, 1987)。Haskett 等 (1997) 应用 GLYCIM 模型对 $\mathrm{CO}_{2}$ 增加条件下的大豆产量进行模 拟也认为大豆产量会增加。虽然人们已经广泛地认 为持续增加空气中 $\mathrm{CO}_{2}$ 量将通过提高植物的光合速 率而使生物量提高, 但在植物之间存在着很大的差 异。长期的 $\mathrm{CO}_{2}$ 加富试验表明: 豆科植物和非豆科 植物对 $\mathrm{CO}_{2}$ 的反应不同, 非豆科植物仅有微弱的地 上生物量的增加, 而豆科植物却反应比较强烈 (Lüscher \& Hebeisen, 1996; Hebeisen, 1997)。

豆科与非豆科植物对 $\mathrm{CO}_{2}$ 浓度升高的响应差异 可以从以下几个方面来解释。首先从源-库关系上 进行分析, 提高“库”的需求可以使光合速率升高 (Bagnall et al., 1988), 与非豆科植物相比, 豆科植 物要将其光合产物的 $1 / 3$ 用作 $\mathrm{N}_{2}$ 的固定和合成、碳 骨架和还原剂 ( Minchin \& Summerfield, 1981)。 $\mathrm{CO}_{2}$ 浓度倍增条件下, 根瘤这个吸收碳同化产物的库容 量增大, 反过来刺激豆科植物叶片进行光合作用, 从 而生产更多的光合产物。对桤木 (Alnus rubra) 的试 验结果也表明, $\mathrm{CO}_{2}$ 浓度倍增条件下将提高同化产 物, 同时也促进了这些同化物向“库” (比如根瘤)的 输出 (Arnone \& Gordon, 1990)。因此, 有自生固氮能 力的 $\mathrm{C}_{3}$ 植物种比其它 $\mathrm{C}_{3}$ 植物对 $\mathrm{CO}_{2}$ 升高的响应更 强烈, 其光合速率的增加也更显著 (Pooter, 1993)。

另外,氮素营养影响着植物对 $\mathrm{CO}_{2}$ 浓度升高的 响应(Pooter \& Roumet, 1996)。 $\mathrm{CO}_{2}$ 浓度增加可以引 起植物光合的提高, 生物量的增加, 但这些变化是在 有充足氮供应的前提下才能形成的, 氮的不足将限 制植物对 $\mathrm{CO}_{2}$ 倍增的响应 (Kramer, 1981)。在自然 
生态系统中, 营养元素尤其是氮素是贫乏的, 这可能 引起植物之间竞争的加剧, 结果导致生态系统中植 物组成的改变 (Bazzaz, 1992; Newton et al., 1994)。 在此竞争过程中, 豆科植物利用其自生固氮能力来 满足对增强了的氮的需求, 从而显示出比非豆科植 物的优越性。即使土壤中的氮素不贫乏, $\mathrm{CO}_{2}$ 的上 升也会使植株个体以至于生态系统对氮的需求增强 ( Ingestad, 1982), 从而造成土壤中有效氮的数量降 低, 反过来又限制了植物的生长和对 $\mathrm{CO}_{2}$ 浓度升高 的反应(Hartwig \& Zanetti, 1996)。在这种情况下, 豆 科植物同样可以通过自身的固氮能力来满足对氮的 需求。

再者, $\mathrm{CO}_{2}$ 浓度升高条件下植物体残留物的 $\mathrm{C} / \mathrm{N}$ 比增大 (Owensby et al., 1993), 从而造成土壤中微 生物的生物量受氮限制, 分解速率降低 (Ginkel et $a l$, 1996; 林伟宏等, 1999), 影响土壤养分的有效 性。但 Soussana 和 Hartwig (1996) 认为豆科植物由 于根瘤菌固氮能力的增加, 使得其调落物中 $\mathrm{C} / \mathrm{N}$ 变 化不大, 因此能够保持正常的分解速率, 保证了光合 和生长的正常进行。

总之, 不管土壤中氮的有效性如何, 豆科植物都 可以通过氮的固定来满足自身因 $\mathrm{CO}_{2}$ 倍增所引起的 对增加了的氮的需求, 保持生态系统中的碳、氮平衡 和元素循环的正常进行, 从这方面来讲, 豆科植物将 比非豆科植物更适应生境中 $\mathrm{CO}_{2}$ 浓度的增加。

$2 \mathrm{CO}_{2}$ 浓度升高对豆科植物固氮特性的影 响

\section{1 对固氮量及固氮活性的影响}

$\mathrm{CO}_{2}$ 浓度升高情况下, 豆科植物自生固氮能力 的变化与地上生物量的增加是一致的。Stulen 和 Hertog (1993)认为, 根瘤固氮能力升高的方式主要 有以下几种: 1) 通过增加植物根瘤数量和根瘤重 量；2）通过增加还原和同化氮所需的能量来源；3） 通过降低矿质氮素吸收来减少植物同化氮时对碳水 化合物的需要。前两种方式会导致植物产量和总固 氮能力的增加, 后一种方式可能使总氮产量的共生 组织增加。

升高 $\mathrm{CO}_{2}$ 浓度提高植物总固氮能力的情形比较 复杂, 结论也不一致。在木本树种洋槐 (Robinia pseudoacasia)、桤木 ( Alnus formosana)（Norby，1987） 以及丁香 ( Syringa oblata) ( Thomas et al., 1991) 中, 比固氮活性（SNA）没有增加, 但根瘤重量和（或） 根瘤数量增加, 表现出每株植物总固氮活性（TNA）
的增加。Arnone 和 Gordon（1990）在桤木的研究中 没有观察到根瘤重量的增加, 但 SNA (比固氮活性) 有所增加, 从而表现出 TNA (总固氮活性) 增加。 David 等(1997)认为高 $\mathrm{CO}_{2}$ 浓度情况下, 虽然 SNA 和 TNA 得以提高, 且固定的氮量也增加, 但在收获期单 株植物的总根瘤量并没有增大。O'N Neil (1994)在对 根瘤的研究中发现, $\mathrm{CO}_{2}$ 浓度增加使草本或木本豆 科植物的单株固氮活性增加, 根瘤重量或数量增加, 但对比固氮活性的影响较为复杂。Pillips (1976) 研 究了踠豆( Pisum sativum) 对瞬时 $\mathrm{CO}_{2}$ 浓度增加的反 应, 发现其比固氮活性倍增, 但经一段时间后, 这种 效应减弱, 长期反应的结果是单株植物总固氮活性 提高。在大豆上做试验也得到类似的结果。丁莉等 (1996) 对 $\mathrm{CO}_{2}$ 浓度增加条件下苜宿个体的固氮能力 变化作了研究, 结果表明其总固氮能力明显得以提 高, 但是比固氮活性与当前 $\mathrm{CO}_{2}$ 条件下的并无多大 差别。对于比固氮活性在 $\mathrm{CO}_{2}$ 倍增条件下变化的复 杂性, 白克智等 (1996) 给出了合理的解释: 单株固 氮活性的提高或来源于根系生物量以及相应根瘤量 的增大, 或来源于根瘤量增加及比固氮活性的增加, 比固氮活性反映了固氮酶催化活性的强弱, 它取决 于 ATP 及 NADPH 的供应水平以及移走铵离子所需 碳架的多少, 这三者都直接或间接来源于光合作用, 而三者的多寡取决于植物生长状态及环境条件。在 $\mathrm{CO}_{2}$ 浓度倍增时, 只有这三者的供应都增强了, 其比 固氮活性才能提高。

\section{2 对其它草类乃至整个生态系统的氮贡献}

从豆科植物向邻近牧草转移氮素的潜在途径 有: 从根系及根瘤中氮的分泌、根和根瘤的衰老及降 解、豆科植物根系与其它牧草之间通过菌根的直接 联系、豆科植物中氨态氮的挥发及被其它牧草直接 吸收利用。在放牧草场, 固定的氮还可以动物粪便 的形式通过地上部分传输给其它草类。在集中放牧 的地方, 豆科植物所固定的氮中大部分被牲畜所消 耗掉, 并且这部分氮中有 $85 \% \sim 95 \%$ 的氮以动物粪 便和尿的形式归还给生态系统。据估计在白三叶和 其它牧草混播草地上, 所固定的氮量为 $269 \mathrm{~kg} \mathrm{~N} \cdot$ $h m^{-2} \cdot \mathrm{a}^{-1}$, 其中通过地上部分向其它草进行传输的 估计为 $60 \mathrm{~kg} \mathrm{~N} \cdot \mathrm{hm}^{-2} \cdot \mathrm{a}^{-1}$, 通过地下部分传输的为 $70 \mathrm{~kg} \mathrm{~N} \cdot \mathrm{hm}^{-2} \cdot \mathrm{a}^{-1}$ 。这样其它草所需要的氮中有 $50 \%$ 可以从白三叶所固定的 $\mathrm{N}_{2}$ 中得到 (Ledgard, 1991)。

豆科植物固氮量的增加不仅对自身有利, 而且 还可以转移给其它不能固氮的植物和有机体。在 
$\mathrm{CO}_{2}$ 浓度增加的条件下, 向其它植物转移氮的比例 也增大（Hebeisen, 1997)。有研究表明, 在 $\mathrm{CO}_{2}$ 浓度 倍增情况下, 白三叶的生物量以及在草地中的比例 均得以增加, 固氮量也增加, 通过地下部分向邻近植 物传输的氮量也增加 (Zanetti \& Hartwig, 1996), 与 其它草类混播的白三叶在高 $\mathrm{CO}_{2}$ 浓度环境下其氮产 量要显著地高于现有 $\mathrm{CO}_{2}$ 浓度条件下, 而且发现在 豆科植物根系中积累的氮并不是来自土壤中的氮而 是完全由生物固定下来的（Schlesinger，1991）。用 ${ }^{15} \mathrm{~N}$ 同位素标记作试验发现混播草地上 $60 \%$ 的氮素 营养来自于豆科植物所固定的氮，在 $\mathrm{CO}_{2}$ 增高条件 下,这种贡献率会更高 (Pooter，1993)。以 Lolium perenne 作试验发现当其单独生长在高 $\mathrm{CO}_{2}$ 浓度下, 其氮获取量下降(生长响应受氮限制), 而与白三叶 共建的群落其年氮获取量增加, 这部分增加的氮源 来源于白三叶的固氮作用(Zanetti \& Hartwig, 1997)。 这说明自然生态系统对 $\mathrm{CO}_{2}$ 浓度升高的响应直接决 定于豆科植物的有无和占多大比例。固氮植物可以 说是草原维持碳与氮平衡的关键因素, 它的存在对 整个生态系统的 $\mathrm{CO}_{2}$ 正反应起着十分重要的作用 (Chapin et al. , 1994)。另外, 草地生态系统的稳定 性和功能也会受 $\mathrm{CO}_{2}$ 浓度的上升而引起的元素循环 所影响。根瘤的作用是改善土壤养分及水分状况， 增加对病原菌和有毒物质的抵抗力, 同时也可作为 光合产物的碳库，因而与根瘤菌共生的植物更有可 能受到高 $\mathrm{CO}_{2}$ 浓度的促进。这样一来, 生态系统中 共生及非共生植物间的关系将有可能改变。从长远 来考虑, 这些变化将会在草地生态系统的结构和生 产力上所体现出来 (Mooney \& Drake, 1991)。

总结前人对 $\mathrm{CO}_{2}$ 浓度增加条件下固氮特性的变 化的研究可以得出如下结论: $\mathrm{CO}_{2}$ 浓度升高使固氮 植物的固氮量增加, 对生态系统的氮素贡献也加大, 但在众多研究中, 由于实验条件和实验对象的差异 而使结果出现不一致现象，因此有待于在此方面进 行更深入、细致的研究。

\section{3 对碳、氮代谢关系的影响}

碳氮代谢是植物最基本的代谢过程，二者密不 可分。豆科植物是个共生体, 寄主植物通过源源不 断地向根瘤输入光合产物而使其固氮功能得以实 现，同时根瘤菌所固定的氮又不断地输向地上部分 供光合需求,在未来全球变化条件下,两者的关系是 否会发生变化?

首先看一下 $\mathrm{CO}_{2}$ 增加条件下, 碳代谢对固氮作
用的影响。在植物吸收所固定的氮之前, 氮必须转 化为有用的形式, 这个转化是由植物的一种酶形成 的, 谷氨酸合成酶就是转化的初始酶, 在 $\mathrm{N}_{2}$ 合成的 功能细胞内, 谷氨酸合成酶得以积累, 而且发现它存 在于细胞的造粉体内, 造粉体中谷氨酸合成酶的发 现说明 $\mathrm{N}_{2}$ 的合成与碳代谢紧密联系在一起, 因为造 粉体是淀粉形成的场所, 这说明生物学家要想提高 或改变 $\mathrm{N}_{2}$ 的合成就必须考虑碳代谢的伴随变化 （Allen et al.，1988）。另外,土壤微生物对生态系统 的影响也受到碳源的限制, 有人曾经提出 $\mathrm{CO}_{2}$ 浓度 升高将给根际微生物带来更多的底物, 从而提高土 壤微生物活性及调落物分解速率, 加速养分的矿化 过程, 改善植物养分状况, 从而使竞争有利于植物, 在此过程中, 固氮菌和菌根菌的作用显得尤为重要 （Ginkel et al., 1997)。在固氮菌进行生物固氮的过 程中, 寄主植物供给共生微生物碳水化合物, 由于碳 水化合物的供应是固氮的一个限制因子, 影响寄主 植物碳固定的因素也将影响固氮过程 (González et $a l ., 2001) 。 \mathrm{CO}_{2}$ 浓度升高增加了植物体的光合产 物, 也增加了光合产物向根瘤的分配, Tissue 和 Megonigal (1997) 对一种热带豆科树种 (Gliricida sepeium) 的研究表明: 在 $\mathrm{CO}_{2}$ 浓度增加条件下, 其光 合产物向根瘤的运输速率加快, 根瘤供碳的增加提 高了比固氮酶活性及总活性, 使固氮量增加, 反过来 又可以使寄主植物受益, 两者相互促进。David 等 (1997) 在生长箱中用 ${ }^{14} \mathrm{CO}_{2}$ 作试验, 发现在 $\mathrm{CO}_{2}$ 浓度 升高情况下, 叶片中的光合产物向根部运输增多, 且 运输速度加快; 比固氮酶活性和总固氮酶活性均提 高, 从而引起固氮量的增加, 另外比固氮酶活性增加 的 $23 \%$ 与向根部供应的光合产物所增加的 $23 \%$ 是 对应一致的, 这说明在碳的供应和固氮酶活性两者 之间的确存在着直接的联系。

根瘤菌的固氮作用对寄主豆科植物光合产物的 依赖程度因种类而异, 对一年生豆科植物而言, 大约 $1 / 3$ 的光合产物被用做 $N_{2}$ 的固定和合成、碳骨架和 还原剂 (Minchin \& Summerfield, 1981)。光合产物形 成之后在不到 $1 \mathrm{~h}$ 时间内就运输到根瘤中。然而, 对于多年生豆科植物而言, 咜存在根中的非结构性 碳水化合物在生长期内也对根瘤有作用 ( Fischbeck \& Philips, 1982)。Hartwig 等 (1990)对光合和固氮的 关系作了更深入的研究, 认为刚合成的光合产物而 不是咜存的光合产物对固氮重要。同时被根瘤 PEPcase (磷酸烯醇式丙酮酸羧化酶) 所固定的 $\mathrm{CO}_{2}$ 也可以提供给根瘤一部分碳源, 这部分碳源在苜宿 
中大约占用于固氮的总碳架的 $12 \%$ 。对大豆来说， 根瘤中大约有一半的 $\mathrm{CO}_{2}$ 通过 PEPcase 来循环 (Warembourg \& Roumet, 1989), 低的根际 $\mathrm{CO}_{2}$ 浓度导 致固氮酶活性的急剧下降。

氮对碳代谢的促进作用在豆科植物上体现得更 明显。氮是 RuBPcase 的主要成分, 催化 $\mathrm{CO}_{2}$ 合成碳 水化合物, 在豆科植物的生长后期也就是籽粒充实 期, 叶片的光合速率往往下降, 但只要保证后期根瘤 菌的固氮能力就可使叶片继续维持高的光合速率 （Salon et al., 2001)。另外, 光合产物的供给是根瘤 菌的生命源泉, 有了它, 固氮酶才有能量进行固氮。 因此, 豆科植物的固氮酶活性既可被碳所调节, 又受 到氮的影响, 这些因素通常包括: 光合、氮的有效性 和对氮的需求 (Hartwig \& Nosberger, 1994; Hunt \& Layzell, 1993)。在 $\mathrm{CO}_{2}$ 浓度升高情况下, 氮的不足 会限制 $\mathrm{CO}_{2}$ 浓度升高对光合的促进, 由此可见氮对 碳代谢的重要性。

$\mathrm{CO}_{2}$ 浓度升高条件下, 既然豆科植物的碳、氮代 谢均受到促进, 那么其 $\mathrm{C} / \mathrm{N}$ 及在器官中的分配如何 呢? 对紫花苜宿的实验结果表明, 地上器官的 $\mathrm{C} / \mathrm{N}$ 增加, 而根系却与之相反 (丁莉等, 1996)。 $\mathrm{CO}_{2}$ 倍增 导致地下部分 $\mathrm{C} / \mathrm{N}$ 的减小, 一个可能的原因是其根 系生物固氮(或)根系吸收氮素的能力比对照更强, 另一个可能的原因是根系固定和吸收的氮素向地上 部的运输受阻使得氮素在根部滞积。在大豆上做实 验也发现类似的结果 (Soussana \& Hartwig, 1996; 白 克智等, 1996)。根系含氮量更高也暗示在 $\mathrm{CO}_{2}$ 倍增 的情况下, 豆科植物可能比非固氮植物具有更高的 增产潜能, 从 Swix FACE 的试验数据上也可以清楚 地看到这一点, 白三叶在 $\mathrm{CO}_{2}$ 加富环境下产量显著 提高, 叶片含氮量下降不明显, 但其它非固氮植物的 产量只有微弱的提高, 叶片含氮量却急剧下降 (Lûscher \& Hebeisen, 1996)。在 INRA 草场实验中也 发现白三叶的叶片氮含量的下降比其它多年生草少 得多 (Casella et al . 1996)。 $\mathrm{CO}_{2}$ 浓度升高之后并非 所有形式的碳水化合物含量都得到增加, 其中可溶 性糖含量就维持在原有水平 $\left(26 \mathrm{~g} \bullet \mathrm{kg}^{-1}\right)$, 淀粉含量 得以明显增加 (Finn \& Brun, 1982)。所增加的碳水 化合物含量也并非均匀地分配到各个器官。接种根 瘤菌 (47 d) 的 Alnus rubra 比不接种的在 $\mathrm{CO}_{2}$ 倍增条 件下, 虽然其叶面积、光合速率和固氮能力均增强, 但其同化产物更倾向于地上部分分配 ( Arnone \& Gordon, 1990)。短期 $\mathrm{CO}_{2}$ 升高(36 h) 所引起的非结 构性碳水化合物的增加主要以淀粉的形式分配于叶
片和叶柄中, 长期加富 ( $16 \mathrm{~d}$ ) 使茎、根和根瘤的干物 重分别增加 $109 \%$ 、34\%和 $56 \%$, 总根瘤活性也得到 提高。这说明 $\mathrm{CO}_{2}$ 加富之后, 地上部分的旺盛生长 才引起了根和根瘤的生长加快 (Finn \& Brun, 1982)。

总之, $\mathrm{CO}_{2}$ 浓度的升高直接导致叶片中光合产 物向根瘤输送的增加, 增加了的碳同化物对 SNA 和 TNA 产生刺激。受到刺激(促进)了的 SNA 和 TNA 增加了整株植物的氮量, 因此促进了 $\mathrm{CO}_{2}$ 浓度增加 条件下的植株的生长, 即使在没有外源氮的情况下 也如此。

综上所述, 前人对于 $\mathrm{CO}_{2}$ 增加条件下豆科植物 的光合生理、固氮特性及其之间的关系进行一定的 研究, 但这些研究只是停留在表面上, 仅仅对 $\mathrm{CO}_{2}$ 浓 度升高后各指标的变化作出描述性的研究, 对 $\mathrm{CO}_{2}$ 的促进效应缺乏生理生化机理方面的探索, 且这些 研究只局限于少量的栽培种, 对于自然生态系统中 的野生豆科植物则几乎无报道, 因此对 $\mathrm{CO}_{2}$ 增加条 件下豆科植物的响应的研究还有待于向深层机理方 面和更广泛的研究对象上发展。

\section{参 考 文 献}

Alberto, A. M., L. H. Ziska, C. R. Cervancia \& P. A. Mahalo. 1996. The influence of increasing carbon dioxide and temperature on competitive interactions between a $\mathrm{C}_{3}$ crop, rice (Oryza sativa) and a $\mathrm{C}_{4}$ weed (Echinocloa glabreseens). Australian Journal of Plant Physiology, 23:795 805.

Allen, L. H., J. C. V. Vu, R. R. Valle, K. J. Boote \& P. H. Jones. 1988. Nonstructural carbohydrates and nitrogen of soybean grown under carbon dioxide enrichment. Crop Science, 28:84 94.

Allen, L. H. \& K. J. Boote. 1987. Response of vegetation to rising carbon dioxide: photosynthesis, biomass and seed yield of soybean. Global Biogeochemistry Cycles, 1:1 14.

Arnone, J. R. \& J. C. Gordon. 1990. Effect of nodulation, nitrogen fixation and $\mathrm{CO}_{2}$ enrichment on the physiology, growth and dry mass allocation of seedlings of Alnus rubra Bong. New Phytologist, 116:55 66.

Bagnall, D. J., R. W. King \& G. D. Farquhar. 1988. Temperature-dependent feedback inhibition of photosynthesis in peanut. Planta, 175:348 354 .

Bai, K. Z. (白克智), Z. P. Zhong (钟泽璞), L. Ding (丁莉), C. H. Zhang (张崇浩), S. Y. Li (李世仪) \& T. Y. Kuang (匡廷云). 1996. Effect of atmospheric $\mathrm{CO}_{2}$ enrichment on some physiological traits in soybean. Chinese Science Bulletin (科学 通报)，41:164 166. (in Chinese)

Bazzaz, F.A. 1992. Plant-plant interactions in elevated $\mathrm{CO}_{2}$ environments. Australian Journal of Botany, 40:547 563 .

Brun, W. A. \& R. L. Cooper. 1967. Effects of light intensity and carbon dioxide concentration on photosynthetic rate of soybean. Crop Science, 7:451 454.

Carter, T. R. 1996. Developing scenarios of atmosphere, weather 
and climate for the northern regions. Agriculture and Food Science in Finland, 5:235 249.

Casella, E., J. F. Soussana \& P. Loiseau 1996. Long-term effects of $\mathrm{CO}_{2}$ enrichment and temperature increase on a temperate grass sward. I. Productivity and water use. Plant and Soil, 182:83 99.

Chapin, F. S., L. R. Walker, C. L. Fastie \& L. C. Sharman. 1994. Mechanisms of primary succession following deglaciation at Glacier Bay, Alaska. Ecological Monographs, 64: 149 175 .

Chen, D. X., H. W. Hunt \& J. A. Morgan. 1996. Response of a $\mathrm{C}_{3}$ and $\mathrm{C}_{4}$ perennial grass to $\mathrm{CO}_{2}$ enrichment and climate change. Ecological Modelling, 87:11 27 .

Clough, J. M., M. M. Peet \& P. J. Kramer. 1981. Effects of high atmospheric $\mathrm{CO}_{2}$ and sink size on rates of photosynthesis of a soybean cultivar. Plant Physiology, 67:1007 1010 .

David, T. \& T. J. P. Megonigal. 1997. Nitrogenase activity and $\mathrm{N}_{2}$ fixation are stimulated by elevated $\mathrm{CO}_{2}$ in a tropical $\mathrm{N}_{2}$-fixing tree. Oecologia, 109:28 33.

Ding, L. (丁莉), Z. P. Zhong (钟泽璞), S. Y. Li (李世仪), C. H. Zhang (张崇浩), K. Z. Bai (白克智) \& T. Y. Kuang (匡廷云). 1996. Assimilation and allocation of carbon and nitrogen in alfalfa under doubled $\mathrm{CO}_{2}$ enrichment. Acta Botanica Sinica (植物学报), 38:83 86. (in Chinese with English abstract)

Drake, B. G., M. A. G. Meler \& S.P. Long. 1997. More efficiency plants: a consequence of rising atmospheric $\mathrm{CO}_{2}$. Annual Review of Plant Physiology and Plant Molecular Biology, 48:609 $\sim 639$.

Finn, G. A. \& W. A. Brun. 1982. Effect of atmospheric $\mathrm{CO}_{2}$ enrichment on growth, nonstructural carbohydrate content, and root nodule activity in soybean. Plant Physiology, 69:327 331 .

Fischbeck, K. A. \& D. A. Phillips. 1982. Host plant and rhizobium effects on acetylene reduction in alfalfa during regrowth. Crop Science, 22:251 254 .

Genthon, C., J. M. Barnola \& D. Raynaud. 1987. Vostok ice core: climatic response to $\mathrm{CO}_{2}$ and orbital forcing changes over the last climatic cycle. Nature, 329:414 418 .

Ginkel, J. H. V., A. Gorissen \& J. A. V. Veen. 1996. Longterm decomposition of grass roots as affected by elevated atmospheric carbon dioxide. Journal of Environmental Quality, 25: $1122 \sim 1128$.

Ginkel, J. H. V., A. Gorissen \& J. A. V. Veen. 1997. Carbon and nitrogen allocation in Lolium perenne in response to elevated atmospheric $\mathrm{CO}_{2}$ with emphasis on soil carbon dynamics. Plant and Soil, 188:299 308.

González, E. M., L. Galez, R. Mercedes, P. M. Aparicio-Tejo \& C. Arrese-legor. 2001. Insights into the regulation of nitrogen fixation in pea nodules: lessons from drought, abscisic acid and increased photoassimilate availability. Agronomie, 21: 607 613.

Hartwig, U. A. \& J. Nosberger. 1994. What triggers the regulation of nitrogenase activity in forage legume nodules after defoliation? Plant and Soil, 161: 109 114.

Hartwig, U. A. \& S. Zanetti. 1996. Symbiotic nitrogen fixation: one key to understand the response of temperate grassland ecosystems to elevated $\mathrm{CO}_{2}$. In: Bazzaz, F. \& C. Körner eds. Carbon dioxide, populations and communities. San Diego: Academic
Press. $253 \sim 264$.

Hartwig, U. A., B. C. Boller \& J. Nosverger. 1990. The influence of carbohydrate reserves on the response of nodulated white clover to defoliation. Annals of Botany, 65:97 105 .

Haskett, J. D., Y. A. Pachepsky \& A. Basil. 1997. Increase of $\mathrm{CO}_{2}$ and climate effects on Iowa soybean yield simulated using GLYCIM. Agronomy Journal, 89: $167 \sim 176$.

Hebeisen, T. 1997. Growth response of Trifolium repens L. and Lolium perenne $\mathrm{L}$. as monocultures and bi-species mixture to free air enrichment and management. Global Change Biology, 3: 149 $\sim 160$.

Hunt, S. \& D. B. Layzell. 1993. Gas exchange of legume nodules and the regulation of nitrogenase activity. Annual Review of Plant Physiology and Plant Molecular Biology, 44:483 511 .

Ingestad, T . 1982. Relative addition rate and external concentration driving variables used in plant nutrition research. Plant, Cell and Environment, 5:443 453.

Kramer, P. J. 1981. Carbon dioxide concentration, photosynthesis and dry matter production. BioScience, 31:29 33 .

Ledgard, S. F.1991. Transfer of fixed nitrogen from white clover to associated grasses in swards grazed by dairy cows estimated using ${ }^{15} \mathrm{~N}$ methods. Plant and Soil, 131:215 223 .

Lin, W. H. (林伟宏), F. S. Zhang (张福锁) \& K. Z. Bai (白 克智). 1999. Effect of atmospheric $\mathrm{CO}_{2}$ enrichment on rhizosphere microecosystem. Chinese Science Bulletin (科学通报), 44: $1690 \sim 1696$. (in Chinese)

Lûscher, A. \& T. Hebeisen. 1996. Differences between legumes and nonlegumes of permanent grassland in their response to freeair carbon dioxide enrichment: its effect on competition in a multispecies mixture. In: Bazzaz, F. \& C. Körner eds. Carbon dioxide, populations and communities. San Diego: Academic Press. $287 \sim 300$.

Minchin, F. R.\& R. J. Summerfield. 1981. Carbon and nitrogen nutrition of nodulated roots of grain legumes. Plant, Cell and Environment, 4:5 16.

Mooney, H. A.\& B. G. Drake. 1991. Predicting ecosystem responses to elevated $\mathrm{CO}_{2}$ concentrations: what has been learned from laboratory experiments on plant physiology and field observation? Biology Science, 41:96 104.

Newton, P. C. D., H. Clark, C. I. Bell, E. M. Glasgow \& B. D. Campbell. 1994. Effects of elevated $\mathrm{CO}_{2}$ and simulated seasonal changes in temperature on the species composition and growth rates of pasture turves. Annals of Botany, 73:53 59.

Norby, R. J. 1987. Nodulation and nitrogenase activity in nitrogenfixing woody plants stimulated by $\mathrm{CO}_{2}$ enrichment of the atmosphere. Physiologia Plantarum, 71:77 82.

O'Neil, E. G. 1994. Response of soil biota to elevated atmospheric carbon dioxide. Plant and Soil, 165:55 65 .

Owensby, C. E., P. I. Loyne \& L. M. Auen. 1993. Nitrogen and phosphorus dynamics of a tallgrass ecosystem exposed to elevated carbon dioxide. Plant, Cell and Environment, 16: 843 850 .

Phillips, D. A. 1976. The effect of $\mathrm{CO}_{2}$ enrichment on root nodule development and symbiotic $\mathrm{N}_{2}$ fixation in Pisum sativum $\mathrm{L}$. American Journal of Botany, 63:356 362 .

Pooter, H. 1993. Interspecific variation in the growth response of plants to an elevated ambient $\mathrm{CO}_{2}$ concentration. Vegetatio, 104/ 
105: $77 \sim 79$.

Pooter, H. \& C. Roumet. 1996. Interspecific variation in the growth response of plants to elevated $\mathrm{CO}_{2}$ : a search for functional types. In: Bazzaz, F. \& C. Körner eds. Carbon dioxide, populations and communities. San Diego: Academic Press. 375 412 .

Ramachandra, R.A., K. R. Reddy, J. M. McKinion \& H. F. Hodges. 1993. $\mathrm{CO}_{2}$ enrichment and temperature effects on the carbon assimilation and transpiration in cotton leaves. Plant Growth Regulation, 26: $33 \sim 40$.

Sage, R. F., T. D. Sharkey \& J. R. Seemann. 1989. Acclimation of photosynthesis to elevated $\mathrm{CO}_{2}$ in five $\mathrm{C}_{3}$ species. Plant Physiology, 89:590 596.

Salon, C., N. G. Munier-Jolain \& D. Gerard.2001. Grain legume seed filling in relation to nitrogen acquisition: a review and prospects with particular reference to pea. Agronomie, 21: 539 $\sim 552$.

Schlesinger, W. H. 1994. Biogeochemistry: an analysis of global change. San Diego: Academic Press.

Servaites, J. C., R. S. Torsky \& S. F. Chao. 1984. Diurnal changes in ribulose 1,5-bisphosphate carboxylase activity and activation state in leaves of field grown soybean. Plant Science, 35: $115 \sim 121$.

Soussana, J. F. \& U. A. Hartwig. 1996. The effect of elevated $\mathrm{CO}_{2}$ on symbiotic $\mathrm{N}_{2}$ fixation: a link between the carbon and nitrogen in grassland ecosystems. Plant and Soil, 187:321 332 .

Stulen, L. \& J. D. Hertog. 1993. Root growth and functioning under atmospheric $\mathrm{CO}_{2}$ enrichment. Vegetatio, 104/105:99 115 .

Thomas, R. B., D. D. Richter, H. Ye, P. R. Heine \& B. R. Strain. 1991. Nitrogen dynamics and growth of seedlings of an Nfixing tree (Gliricidia sepium (Jacq. Walp. )) exposed to elevated atmospheric carbon dioxide. Oecologia, 8:415 421.

Tissue, D. T. \& J. P. Megonigal. 1997. Nitrogenase activity and $\mathrm{N}_{2}$ fixation are stimulated by elevated $\mathrm{CO}_{2}$ in a tropical $\mathrm{N}_{2}$-fixing tree. Oecologia, 109:28 33.

Vu, J. C. V.\& L. H. Allen. 1997. Effects of elevated $\mathrm{CO}_{2}$ and temperature on photosynthesis and Rubisco in rice and soybean. Plant, Cell and Environment, 20:68 76.

Warembourg, F. R. \& C. Roumet. 1989. Why and how to estimate the cost of symbiotic $\mathrm{N}_{2}$ fixation? A progressive approach based on the use of ${ }^{14} \mathrm{C}$ and ${ }^{15} \mathrm{~N}$ isotopes. Plant and Soil, 115: $167 \sim 178$.

Webber, A. N., G.Y. Nie \& S. P. Long. 1995. Acclimation of photosynthetic proteins to rising atmospheric $\mathrm{CO}_{2}$. Photosynthesis Research, 39:413 426.

William, J., L. H. Campbell, J. R. Auen \& B. George. 1988. Effects of $\mathrm{CO}_{2}$ concentration on Rubisco activity amount and photosynthesis in soybean leaves. Plant Physiology, 88: 1310 1316.

Xiang, B. (项斌)，S. H. Lin (林舜华) \& L. M. Gao(高雷 明). 1996. Responses to doubled $\mathrm{CO}_{2}$ in Medicage sativa: studies on ecophysiology and simulation modeling. Acta Botanica Sinica(植物学报), 38:63 71. (in Chinese with English abstract)

Yu, Z. W. (余振文) . 1993. Crop physiology. Beijing: China Agricultural Press. $172 \sim 173$.

Zanetti, S. \& U. A. Hartwig. 1997. Does nitrogen nutrition restrict the $\mathrm{CO}_{2}$ response of fertile grassland lacking legumes. Oecologia, 112: $17 \sim 25$.

Zanetti, S. \& U. A. Hartwig. 1996. Stimulation of symbiotic $\mathrm{N}_{2}$ fixation in Trifolium repens $\mathrm{L}$. under elevated atmospheric $\mathrm{CO}_{2}$ in a grassland ecosystem. Plant Physiology, 112:575 583 .

Zhang, Q. D. (张其德), C. M. Lu (卢从明), L. J. Feng (冯 丽洁), S. Y. Li (李世仪), T. Y. Kuang (匡廷云) \& K. Z. Bai (白克智). 1996. Effect of elevated $\mathrm{CO}_{2}$ on the primary conversion of light energy of alfalfa photosynthesis. Acta Botanica Sinica (植物学报), 38:77 82. (in Chinese with English abstract) 